\title{
Cross Species use of Human Microarray Genotyping Technology for Bornean Orangutan (Pongo pygmaeus) SNP Discovery
}

\author{
Ruth Ella Linsky ${ }^{1,2 *}$, R. Steven Wagner ${ }^{1,3}$, Reniastoetie Djojoasmoro ${ }^{4}$, Joseph Lorenz ${ }^{1,5}$, Biruté M. F. Galdikas ${ }^{2,4}$ \\ ${ }^{1}$ Primate Behavior Program, Central Washington University, Ellensburg, USA \\ ${ }^{2}$ Simon Fraser University, Burnaby, Canada \\ ${ }^{3}$ Biotecon Diagnostics, Hermannswerder 17, Potsdam, Germany \\ ${ }^{4}$ Orangutan Foundation International, Jakarta, Indonesia \\ ${ }^{5}$ Eichendorffstrasse 3268167 Mannheim, Germany
}

\section{ARTICLE INFO}

Article history:

Received November 18, 2019

Received in revised form September 30, 2021

Accepted October 19, 2021

\section{KEYWORDS:}

Bornean orangutan,

Pongo pygmaeus,

SNP,

microarray,

Tanjung Puting

\begin{abstract}
Previous genetic studies of orangutans (Pongo spp.) have relied mainly upon mitochondrial DNA or microsatellite short tandem repeats (STR) for genomic genotyping analysis. Scientists have yet to take advantage of the genetic closeness of the great apes to humans for genomic analysis by using advanced techniques available for human genotyping. To genotype orangutans at Tanjung Puting National Park, we developed a novel combination of a methyl-based magnetic enrichment capture of genomic fecal DNA with genotyping on a human targeted single nucleotide polymorphism (SNP) microarray, and compared this to additional microsatellite (STR) micro-capillary genotyping. We successfully isolated 125 known human genomic SNP loci $(0.08 \%$ of those targeted) which hybridized orangutan DNA on the human targeted Illumina Infinium QC array. We estimated genetic diversity and relatedness ( $r$ ) using three estimators for a total of 32 (21 female and 9 male) wild orangutans at the Camp Leakey study site. Average TrioML relatedness within the sample, estimated from our combo SNP/ STR dataset, was at a range consistent with half and first cousins $(r=.082)$. All sampled males and females had relatives within the study site indicating we have verified a local, closely related community of wild orangutans at Camp Leakey.
\end{abstract}

\section{Introduction}

In accordance with regulations surrounding endangered species to avoid invasive sampling practices, geneticists have moved towards noninvasive methods by collecting feces, urine, food waste, and other discarded materials (Inoue et al. 2007; Rutledge et al. 2009). It is now possible to extract viable DNA from these wildlife by-products and primate conservationists have begun to build protocols to investigate populations using these sample types (Goossens et al. 2000; Nsubuga et al. 2004; Simons et al. 2012).

Orangutans (genus: Pongo) as semi-solitary arboreal, deep forest primate group, present an extreme example of how difficult non-invasive genetic sampling can be in the wild. However, over the past two decades there have been several studies using non-invasive fecal sampling investigating

\footnotetext{
* Corresponding Author

E-mail Address: ruth linsky@sfu.ca
}

relatedness and metrics of genetic diversity within populations from study sites in Borneo and Sumatra. These studies which used different methods and genetic markers produced a range of results for each site; for example higher female relatedness was found in Sebangau ( $Q$ and $G r=0.046$ and Wang $r=$ 0.166; Morrogh-Bernard et al. 2011) and in Tuanan (TrioML, Wang, and $Q$ and G; Arora et al. 2012) and more equal but low relatedness within males and females at Ketambe ( $Q$ and $G r=-0.095$ and $r=-0.108$, respectively; Utami et al. 2002) and more equal but high relatedness within males and females at the Lower Kinabatangan Wildlife Sanctuary $(Q$ and $G$ $r=0.142$ and $r=0.148$, respectively, Goossens et al. 2006). Understanding how these relatedness values are influenced by dispersal and reproductive patterns have direct consequences for species viability in the wild. Thus, having high quality and efficient methods to study these patterns is important for assessing management options.

Despite much improvement in genotyping practices, microsatellite (or Short Tandem Repeat, 
STR) analysis used in the majority of past orangutan genotyping studies is time consuming and requires multiple sample replicates to ensure all loci are amplified during the PCR process; further it is often plagued with issues of low initial template DNA and failure of amplification of some loci due to inhibitors from co-extracted fecal matter. STR DNA fragments also require visualization through gel electrophoresis which is another labor intensive multistep process.

Recent improvements in next generation sequencing and genotyping techniques have provided a relatively swift genotyping process which is increasingly affordable for often inadequately funded primate conservation projects (Vigilant and Guschanski 2009). Single nucleotide polymorphism (SNPs) loci have been identified and mapped across the human genome, and are being discovered increasingly for model and non-model organisms (Kanthaswamy et al. 2009; Norman et al. 2013; Rianti et al. 2015; Bourgeois et al. 2018). The use of commercially available sequencing kits and microarray genotyping chips across closely related species has helped discovery of conserved SNP loci (Miller et al. 2011; Ogden et al. 2012; Hoffman et al. 2013). However, researchers have not yet taken advantage of the relative genetic closeness of orangutans to humans for which the most commercial products are currently targeted.

In order to streamline the process of non-invasive genotyping to investigate the genetic relatedness of a previously unsampled local wild Bornean orangutan (Pongo pygmaeus) population at the Camp Leakey research site, we designed and assessed a new protocol for microarray SNP genotyping of orangutan DNA isolated from feces. This paper details a novel process which combines fecal DNA extraction with a modified magnetic bead enrichment capture technique, FecalSeq (Chiou and Bergey 2018), followed by orangutan genomic SNP genotyping by cross species use of human targeted microarray chips. Relatedness estimates produced by genotyping with this new process are complimented and assessed by comparison with a micro-capillary STR marker genotype dataset.

\section{Materials and Methods}

\subsection{Study Site}

With an area of approximately 4,150 $\mathrm{km}^{2}(1,886$ $\mathrm{km}^{2}$ of orangutan habitat), Tanjung Puting National Park (Figure 1) is one of the largest protected areas in Central Kalimantan (Utami-Atmoko et al., 2017). The Camp Leakey study area was initially established within a $35 \mathrm{~km}^{2}$ area and contains a mix of dry ground

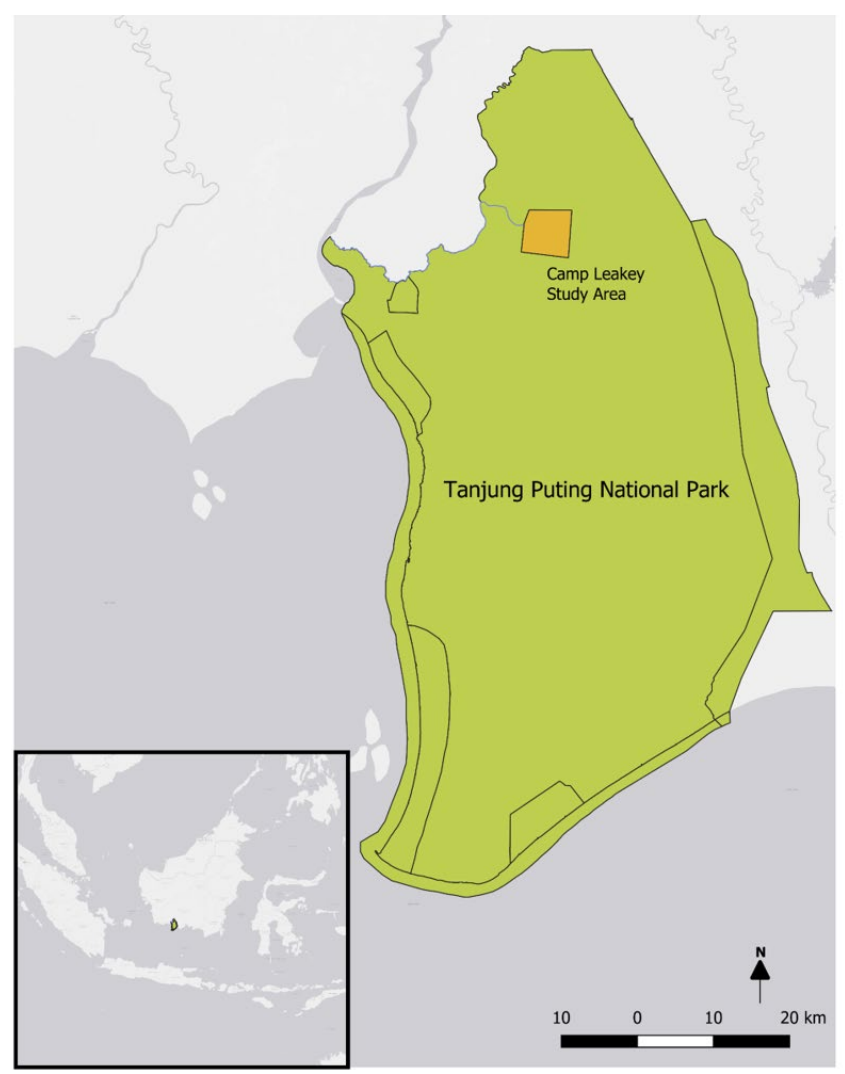

Figure 1. The Camp Leakey Study Area is located along the Sekonyer River in the northern portion of Tanjung Putting National Park in Central Kalimantan, Indonesian Borneo, Indonesia

tropical heath and dipterocarp forests with veins of permanently wet and seasonally flooded peat swamp threaded throughout (Galdikas 1979). The local wild orangutans are behaviorally observed on an ongoing basis within the study area which contains maintained trails (Galdikas 1982, 1985, 1988).

In ongoing data collection at Camp Leakey since its establishment, local wild individuals who are identifiable by local staff and researchers, as well as any unidentified individuals encountered, are behaviorally studied through follows from nest to nest each day. Data are collected by focal follows of orangutans for ten day periods. Matrilineal lines have been recorded for at least three generations on females whose home range included the Camp Leakey study area site. Thus, some relatedness values between sampled individuals are known and mother offspring pairs were identified in Table 1.

\subsection{Sample Collection}

Fecal sampling of wild orangutans within the Camp Leakey study area took place between January and August 2016. Once located and visually identified and 
Table 1. Orangutans genotyped in this study

\begin{tabular}{|c|c|c|c|c|}
\hline SNP and STR & Orangutan ID & Age class & Sex & Previously known relationships \\
\hline SNP and STR & BDM1 (Mooch) & Adult & Female & $\begin{array}{l}\text { BDM1 is mother to JMM12 and } \\
\text { ABDM12 and older sister to BDM3 }\end{array}$ \\
\hline SNP and STR & JMM12 (Mario) & Juvenile & Male & \\
\hline SNP and STR & ABDM12 (Molly) & Infant & Female & \\
\hline SNP and STR & BDM3 (Macey) & Adolescent & Female & Younger sister to BDM1 \\
\hline SNP and STR & BDN1 (Noisy) & Adult & Female & No known \\
\hline SNP and STR & BDN11 & Adult & Female & No known \\
\hline SNP and STR & BDR1 (Renie) & Adult & Female & BDR1 is mother to ABDR1 \\
\hline SNP and STR & ABDR1 (Rutha) & Infant & Female & \\
\hline SNP and STR & BDP1 (Pete) & Adult & Female & BDP1 is mother to BR01 \\
\hline $\mathrm{SNP}^{* *}$ and STR & BR01 (Pamela) & Adolescent & Female & \\
\hline SNP and STR & BD01 (Erma) & Adult & Female & BD01 is mother to ABD01 \\
\hline SNP and STR & ABD01 (E2) & Infant & Female & \\
\hline $\mathrm{SNP}^{* *}$ and STR & BD02 (Aluh) & Adult & Female & No known \\
\hline SNP and STR & BD02Q & Adult & Female & No known \\
\hline SNP and STR & BD04 & Adult & Female & No known \\
\hline SNP and STR & ABD05 & Infant & Male & No known \\
\hline SNP and STR & JDP1 (Ponorogo) & Adult & Male & No known \\
\hline SNP and STR & JD01 & Adult & Male & No known \\
\hline SNP and STR & JR02 & Juvenile/adolescent & Male & No known \\
\hline SNP and STR & JR08 & Juvenile/adolescent & Male & No known \\
\hline SNP and STR & JR09 & Sub adult & Male & No known \\
\hline SNP & JD03 & Sub adult & Male & No known \\
\hline SNP & JR01 & Juvenile/adolescent & Male & No known \\
\hline SNP & ABDM21 (Maureen) & Infant & Female & $\mathrm{ABDM} 21$ is niece to BDM1 and BDM3 \\
\hline STR & BD07 & Adult & Female & $\mathrm{BD} 07$ is mother to $\mathrm{ABD} 07$ \\
\hline SNP and STR & ABD07 & Infant & Female & \\
\hline STR & BD06 & Adult & Female & BD06 is mother to ABD06 \\
\hline STR & ABD06 & Infant & Female & \\
\hline STR & BD03 & Adult & Female & No known \\
\hline STR & AB10 & Juvenile & Female & No known \\
\hline STR & BD08 (Beth) & Adult & Female & No known \\
\hline STR & BD09 & Adult & Female & No known \\
\hline
\end{tabular}

confirmed by experienced field assistants as either a known or unknown wild individual, orangutans were followed continuously until defecation was observed. Fecal samples were collected in duplicate and stored as per a two-step method (Nsubuga et al. 2004). Samples (approx. 2-10 g) were collected using sterile gloves and a sterile collection spoon to avoid contamination, and initially stored in $30 \mathrm{ml}$ of $97 \%$ ethanol solution (step one). Ethanol solution was discarded 24 to 36 hours later and $\sim 10 \mathrm{~g}$ of silica gel beads were placed inside sample container (step two). Samples were then transferred stored in refrigerator at $-40^{\circ} \mathrm{C}$ until processing. Samples were collected under approved Central Washington University IACUC, with permissions from the Indonesian Institute of Sciences (LIPI), the Director general of Indonesian Directorate of Biodiversity Conservation, with prior informed consent from Tanjung Puting National Park with assistance from Orangutan Foundation International staff, and exported from Indonesia to Central Washington University under the Convention on International Trade in Endangered Species export permit 01152/IV/SATS-LN/2017.

\subsection{DNA Extraction}

DNA was extracted using the QIAamp DNA Stool Mini Kit ${ }^{\circledR}$ Qiagen DNA extraction kit. Initial DNA extraction was conducted by hand and then subsequent extractions were automated using the QIAcube $^{\mathrm{TM}}$ robot for increased standardization. The standard kit protocol was used except with modification of an extended cell lysis step with an overnight incubation period of $14-18$ hours in $23^{\circ} \mathrm{C}$ heat block after addition of lysis buffer and prior to 
insertion into the QIAcube ${ }^{\mathrm{TM}}$ machine. An additional extended incubation hold of 30-120 mins was added before final elution step. For microsatellite (STR) analysis, extracts underwent a double inhibitor cleanse where samples (or existing extracts) were incubated in Inhibit-Ex buffer as well as an InhibitEx tablet for the 14-18 hours as stated above. Total DNA was then quantified using a NanoDrop 2000 spectrophotometer reading.

Analysis was conducted on DNA isolated from fecal samples from 32 wild individuals at Camp Leakey. This included two adult wild males, 14 adult females, two adolescent nulliparous females (with known maternity), eight infant or young juvenile offspring (seven with known maternity among those sampled), four juvenile/adolescent males (one with known maternity, the others unknown and found traveling on their own), and two unknown subadult males. In total 15 of these individuals were known and named, in that they were identified by at least two local field staff and witnessed on multiple occasions in the study area during sample collection. The others represent either unknown individuals or those whose identity was not absolutely verified at the time of collection. The orangutans sampled and genotyped in this study are listed in Table 1.

To gauge initial quantity of orangutan DNA extractions, quantitative real-time PCR (qPCR) was conducted on samples using universal mammalian MYCBP primers (Higuchi et al. 1993) and a SYBR green Universal Master Mix on the BioRad iQ5 Optical qPCR system. Multiple (2-4) DNA extractions were conducted for each individual (except three individuals were only able to be extracted once due to a low quantity sample) and initial 69 DNA samples were evaluated for quality and relative quantity by the ability to be amplified with real time PCR. Only samples with total orangutan DNA greater than 20 ng were used for the SNP microarray analysis. Extracts that were less than $20 \mathrm{ng}$ were pooled for each individual for either further enrichment or to be run directly on the microarray. For those individuals whose samples were pooled for SNP genotyping, further extracts were conducted for STR analysis but were not evaluated and quantified using qPCR.

\subsection{Genotyping}

For microarray SNP analysis, the FecalSeq (Chiou and Bergey 2018) technique, based on the New England Bio-labs NEBnext Microbiome DNA Enrichment Kit, was used on a subset of 27 individuals to separate host orangutan DNA from co-extracted fecal microbial DNA. This technique uses methyltagged magnetic beads which bind selectively to
CpG-methylated eukaryotic DNA which can then be separated from the remaining bacterial sample using a magnet. The resulting host enriched DNA was utilized for microarray SNP analysis. Samples post enrichment were further evaluated and quantified using the above outlined qPCR technique, with the addition of a universal bacterial 16S rRNA primer (Corless et al. 2000), to test for a decrease in bacterial concentration in order to assess sample enrichment success.

SNP microarray analysis was conducted on 48 samples which consisted of the following: 1 human DNA extract used as a positive control, 27 extracts from theFecalSeqenrichment process, and the remaining 20 were un-enriched extraction products. DNA extracts underwent a quality check and gender confirmation using Taqman real time PCR quantification and were run on an Illumina Infinium Human QC microarray SNP chip to identify homologous human single nucleotide polymorphism (SNP) loci. Microbeads on the chip hybridize specific known human SNP locations using targeted probes. UV light causes fluorescence of the bound colored probes as specific nucleotides hybridize resulting in light intensity and color data. These data are translated using custom proprietary Illumina software, GenomeStudio2.0.

In order to assess the quality of microarray genotypes and resulting relatedness estimates, microsatellite (STR) marker genotyping through targeted amplification and visualization was also performed. Eight STR autosomal markers were selected from those used in several former studies and described by Nietlisbach et al. (2010). These shortrepeated sections were amplified using targeted primers and through polymerase chain reaction (PCR) thermocycling. PCR conditions followed Arrora et al. (2010) and Nietlisbach et al.(2010) using SigmaAldrich Redtaq mastermix. A subset of samples underwent multiple amplifications for each of the targeted regions. Error rates were calculated from this subset. PCR amplifications were electrophoresed on microcapillary DNA1000 chips on the Agilant Bioanalyzer 2100 machine. Resulting electropherogram data were visualized and analyzed using the Agilant 2100 Expert software. Fragment variant lengths for eight autosomal tetra-nucleotide loci, five Pongo specific (Nietlisbach et al. 2010) and three human specific (Goossens et al. 2005), (Table 1) were coded visually using the gel-like densitometry plot data comparison view. High quality Pongo DNA, and human DNA, as positive control, were amplified alongside samples to confirm band sizes and intensity. Bands were identified as separate loci when repeatedly amplified or observed (more than once) and when at least four 
base pairs apart from bands above or below (once corrections between chip runs were done). In total 29 individuals underwent genotyping using this method (e.g., 22 of the same individuals with SNP genotypes).

\subsection{Statistical Analysis}

Allele frequencies and distinct individual identities were confirmed using Cervus (Kalinowski et al. 2007) software for both genotyping techniques (e.g., microarray and STR). Resulting genotypes from both methods were analyzed for Hardy-Weinberg equilibrium and to assess linkage disequilibrium using online software GenePop (Rousset 2008). In order to assess quality of SNP genotypes and to compare to the STR dataset, resulting pairwise relatedness values were calculated for the 22 individuals within both datasets using the triadic likelihood estimator, TrioML (Wang 2007), and two moment estimators used in past studies, the coefficient of Wang (Wang 2002), and Queller and Goodnight pairwise relatedness estimator (Queller and Goodnight 1989), $r_{x y}$, using the Colony 2.0 (Jones and Wang 2010) and COANCESTRY 1.0 (Wang 2011) software. Relatedness values for the subset of 22 individuals within both datasets were calculated using allele frequencies from adults in each entire dataset. Overall group and pairwise relatedness values calculated with each estimator were tested for correlation through paired and unpaired $t$-tests and Mantel matrix correlation tests in the ade4 (Dray and Dufour 2007) package in the R statistical environment ( $\mathrm{R}$ Core Team 2014).

\section{Results}

\subsection{Genotyping}

Average total orangutan DNA proportion per $100 \mu \mathrm{l}$ extract was $3.31 \%$ (range $<0.01-82.7 \%$ ). After undergoing the FecalSeq magnetic bead enrichment process to separate endogenous orangutan DNA from that of contaminating microbiome found in feces, bacterial DNA quantities in extracts decreased from 30-500 fold (median 140 fold decrease). While Pongo DNA quantities decreased as well, the average was by about half ( median $=57 \%$ initial DNA remaining, range $12-95.8 \%$ ), but was enriched compared to bacterial DNA. The 27 post-enrichment samples with highest orangutan concentrations, were then chosen to be run on the Illumina SNP microarray. Initial quality control Taqman qPCR testing confirmed the known sexes of 38 of 45 samples run on the microarray (with the 7 unconfirmed due to low signal and none providing opposite sex assignment) as well as identified the unknown sex of three infants. Total DNA concentrations for enriched samples ranged from
$0.85 \mathrm{ng} / \mu \mathrm{l}$ to $7.39 \mathrm{ng} / \mu \mathrm{l}$ and total DNA concentrations for un-enriched samples were $14.3 \mathrm{ng} / \mu \mathrm{l}$ to $48 \mathrm{ng} / \mu \mathrm{l}$.

Microarray data from un-enriched samples was poor, with light intensity (Norm R) and color (Norm Theta) results scattered across the spectrum (grey scattered dots in Figure 2). Clustering did not occur as should be expected in comparison to human DNA results. However, for the enriched samples visual inspection identified 125 of the SNP loci out of 15 , 949 on the microarray that provided clear assignment signals and clusters (green dots in Figure 2). These 125 loci $(0.78 \%$ of the total on the microarray) were further identified as presenting similar clustering patterns to those of human data (examples of this at four SNP loci discovered are shown in Figure 2) and presenting high enough minor allele frequencies $(125$ $>0.018,104>0.1,61>0.2$ ) to be used for genotyping.

The 125 homologous bi-allelic SNP loci (Supplementary S1) were used to create genotypes for 27 individuals at a minimum of 65 (52\% of the total 125) loci. Through identity analysis, two pairs of samples originally assumed to represent four unique individuals in the 27 individuals in this SNP dataset were identified as representing two individuals each sampled twice, matching at respectively all 93 and 81 loci in common and both pairs mismatching at 0 loci. These two pairs of individuals were confirmed as representing just two individuals sampled in repetition but whose identities were not $100 \%$ confirmed in the field at the time of collection. The mean proportion of the 125 SNP loci typed for the 27 individuals was 0.72 and combined non-exclusion probability of identity was 3.07e-37.

STR genotyping was conducted on 355 PCR amplifications for 33 individuals. Samples from four individuals repeatedly did not amplify so we were unable to genotype them using this method. Thus, we produced successful genotypes for 29 individuals. Of the successful amplifications 39\% were individuals genotyped in duplicate, $14 \%$ were individuals genotyped in triplicate, and $7 \%$ were individuals genotyped more than three times. Allelic drop out error rates were calculated from this multiple genotyping to be 0.055 . Mean proportion of STR loci typed was 0.88 , and combined non-exclusion probability of identity was $6.6 \mathrm{e}^{-7}$.

\subsection{Genetic Diversity}

Overall average observed heterozygosity (Ho) for the 125 SNP markers was $0.36, \mathrm{SD}=0.19$, average expected heterozygosity (He) was $0.34, S D=0.14$, with average polymorphic information content (PIC) of $0.27, \mathrm{SD}=0.092$. Average inbreeding co-efficient (FIS) was $-0.037, \mathrm{SD}=0.32$. Of the 125 individual SNPs, 

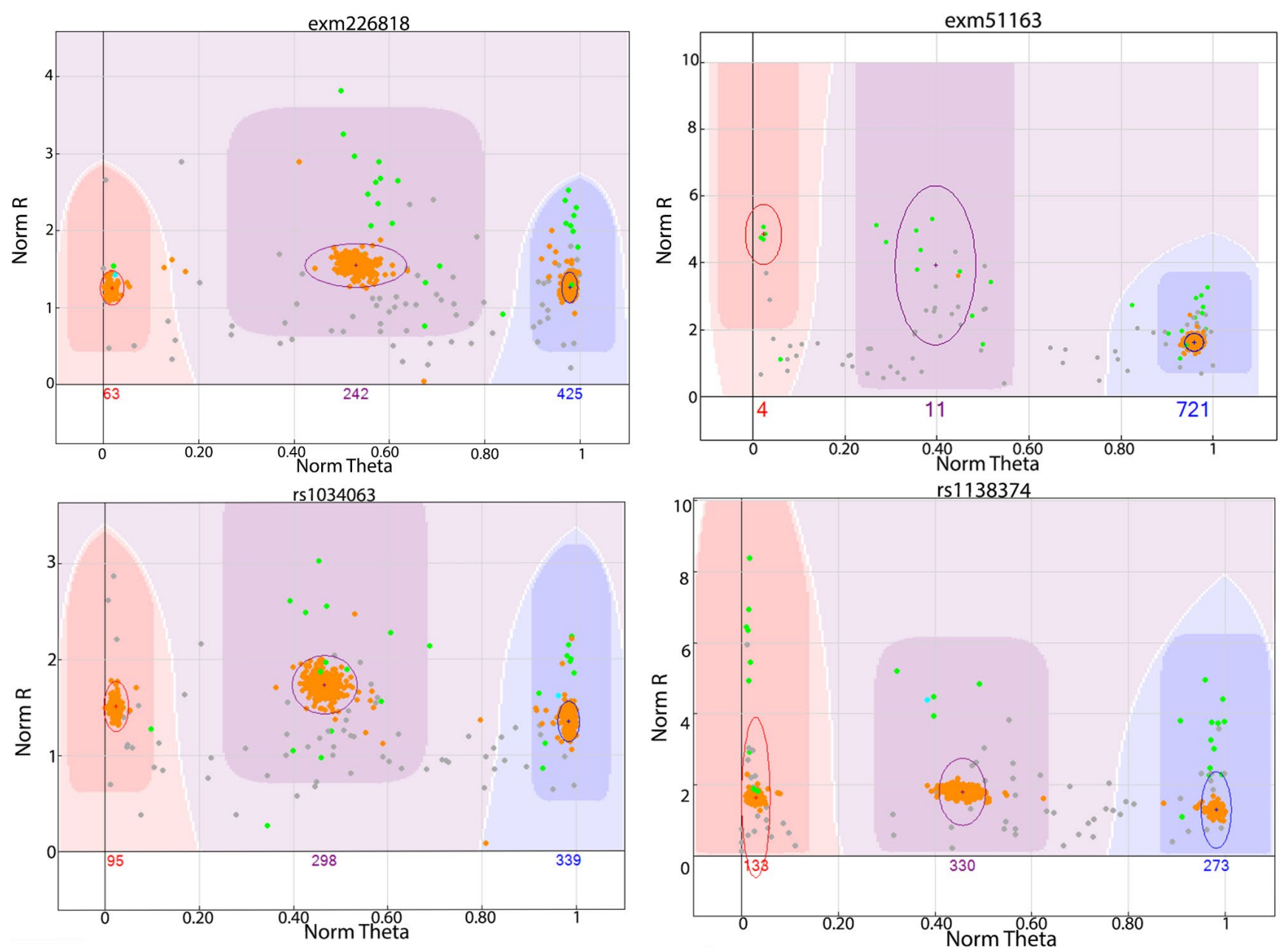

Figure 2. GenomeStudio SNP graph clustering results for four SNP loci. Orange dots are sample human data, green dots are the 27 enriched samples and dark grey dots are unenriched samples. Norm Theta represents the light color read and Norm $\mathrm{R}$ is the light intensity. Circles and dark colouration are areas where allele assignments or calls are made. The two exterior red and blue circles represent homozygous calls and center purple is heterozygous call

10 loci differed significantly from $\mathrm{H}-\mathrm{W}$ equilibrium $(\alpha=0.05)$, with both lower and higher than expected heterozygosity and corresponding high and low FIS values. These values can be found in Supplementary S1.

Allele frequency calculations and tests for heterozygosity and deviation from Hardy-Weinberg (H-W) equilibrium for the eight microsatellite STR autosomal markers found average observed heterozygosity ( $\mathrm{Ho}$ ) to be 0.65 with an expected $(\mathrm{He})$ value of 0.66 with average PIC of 0.591 , with one marker D13S765 showing significant deviation from $\mathrm{H}-\mathrm{W}$ equilibrium $(\mathrm{p}=0.001)$. Average FIS inbreeding coefficient for the 8 autosomal markers was 0.003 $(\mathrm{SD}=0.14)$. These values can be found in Table 2 .

\subsection{Relatedness}

Initial Cervus identity analysis of the SNP dataset also confirmed the shared identity of the two pairs of individuals each sampled twice within the SNP samples as well as verified all other individuals as unique. These duplicate genotypes were removed and not used for further analysis. Identity analysis of the STR data confirmed all the individuals as unique. This resulted in 25 individuals with SNP data, 29 with STR data, and 32 unique individuals in total.

Pairwise relatedness estimates of the coefficient of Wang produced by Coancestry and parentage analysis using Colony confirmed four of five known mother offspring pairs in the SNP Data and the one nonconfirmed pair gave a maximum $r$ estimate of 0.23 , and confirmed all seven within the STR data (Table 3 ). Average mother-offspring relatedness across all three estimators was $0.46 \mathrm{sd}=0.19$ (SNP data) and $0.43 \mathrm{sd}$ $=0.16$ (STR data).

Pairwise relatedness values for the 22 individuals with both SNP and STR genotypes were compared using a Mantel correlation test and showed a positive 
Table 2. Autosomal microsatellite (STR) markers

\begin{tabular}{lccccccccr}
\hline Name & Marker type & $\mathrm{A}$ & $n$ & $\mathrm{H}_{\mathrm{O}}$ & $\mathrm{H}_{\mathrm{E}}$ & PIC & P-val & S.E. & $\mathrm{F}_{\text {IS }}$ Wa and C \\
\hline O4_6 & Pongo & 3 & 25 & 0.60 & 0.49 & 0.42 & 0.652 & 0.004 & -0.233 \\
O4_B5 & Pongo & 5 & 26 & 0.81 & 0.78 & 0.72 & 0.194 & 0.007 & -0.042 \\
O4_A1 & Pongo & 4 & 29 & 0.69 & 0.64 & 0.55 & 0.514 & 0.008 & -0.082 \\
O4_B20 & Pongo & 2 & 25 & 0.40 & 0.44 & 0.34 & 0.663 & 0.002 & 0.101 \\
O4_CHR5 & Pongo & 6 & 26 & 0.77 & 0.69 & 0.64 & 0.850 & 0.007 & -0.112 \\
D13S765 & Human & 6 & 27 & 0.59 & 0.75 & 0.69 & $0.001^{*}$ & 0.001 & 0.213 \\
D6S501 & Human & 7 & 25 & 0.72 & 0.74 & 0.69 & 0.439 & 0.016 & 0.020 \\
D13S321 & Human & 4 & 21 & 0.62 & 0.73 & 0.66 & 0.580 & 0.006 & 0.160 \\
\hline & Mean & 5 & 26 & 0.65 & 0.66 & 0.59 & & & 0.003 \\
& SD & 2 & 2 & 0.12 & 0.12 & 0.13 & & & 0.140 \\
\hline
\end{tabular}

*indicates a statistical departure from HW equilibrium

Allelic diversity A, number of genotyped individuals, observed heterozygosity $\mathrm{H}_{0}$, expected heterozygosity $\mathrm{H}_{\mathrm{E}}$, polymorphic information content PIC, average $\mathrm{F}_{\text {IS }}$ (Weir and Cockerham 1984) and p-value plus standard error S.E. of probability test for deviation from Hardy-Weinberg (HW) equilibrium

Table 3. Expected and estimated pairwise relatedness (Wang $(r)$ ) for known mother-offspring pairs

\begin{tabular}{llccc}
\hline Related mother-offspring Pairs & Expected relatedness & STR relatedness & SNP relatedness \\
\hline BDM1 & JMM12 & $\sim 0.5$ & 0.49 & 0.45 \\
BDM1 & ABDM12 & $\sim 0.5$ & 0.44 & 0.71 \\
BDR1 & ABDR1 & $\sim 0.5$ & 0.48 & 0.23 \\
BDP1 & BR01 & $\sim 0.5$ & 0.67 & 0.61 \\
BD01 & ABD01 & $\sim 0.5$ & 0.38 & 0.64 \\
BD07 & ABD07 & $\sim 0.5$ & 0.57 & - \\
BD06 & ABD06 & $\sim 0.5$ & 0.61 & - \\
\hline & & Average & 0.52 & 0.53 \\
& SD & 0.10 & 0.19 \\
\hline
\end{tabular}

correlation for all three estimators TrioML $(r=0.81, \mathrm{p}$ $<0.001)$, Wang $(r=0.34, \mathrm{p}=0.018)$, and Queller and Goodnight ( $r=0.42, \mathrm{p}=0.0032$ ). The two datasets were then combined for all 32 individuals and a third new "Combo" dataset was produced and compared.

Overall relatedness was calculated for each data set using three estimators and the combined dataset and is presented in Table 2. Overall relatedness in all 32 individuals from the Combo (SNP and STR) dataset using the TrioML estimator was $0.082($ var $=0.021)$.

Results of a paired t-test to compare the overall TrioML averages of the SNP $(r=0.096$, var $=0.023)$ and the Combo dataset found no significant difference with conditions $(t(612)=-1.31, \mathrm{p}=0.19)$. A test to compare the STR mean $r=0.082(v a r=0.020)$ and the Combo dataset also showed no significant difference $(t(869)=0.022, \mathrm{p}=0.98)$.

We determined all males were related to two or more individuals in our sample at the level of first cousins or higher. A mother was able to be assigned to two unknown males (one subadult and one juvenile) within the study area. The two adult fully flanged males were estimated to have first cousin and higher relatedness with both females and other males within the sample.
Average adult female relatedness within the sample is approximately between the levels of half cousin (or first cousin once removed) and first cousins (e.g., $r$ values range $0.0625-0.125$ ), with all adult and juvenile females having a close (e.g., at least half sib, aunt/niece, or first cousin) relative within the study area. One unknown adult female-adult daughter pair observed and sampled within close proximity was identified. This is the same mother of the two unknown males. Two other unknown adult females were identified as full siblings.

\section{Discussion}

This is the first known study to combine the use of non-invasive fecal DNA sampling and extraction, methyl based enrichment FecalSeq ${ }^{\mathrm{TM}}$ (Chiou and Bergey 2018), and human targeted Illumina Infinium SNP microarray genotyping technology for population monitoring of an endangered great ape. The use of fecal DNA sampling has become standard for cryptic and sensitive endangered species. However, difficulties arising from low endogenous DNA quantity within samples remain 
pervasive. Combining extraction with the FecalSeq methyl based magnetic bead capture enrichment technique increased the concentration of orangutan DNA (vs bacterial DNA etc). Of the samples that were tested on the Illumina microarray chip only those that underwent the FecalSeq enrichment process produced successful genotypes. Initial quantification of DNA samples indicated averages of approximately $3 \%$ endogenous DNA in our fecal extractions which is consistent with published numbers (Perry et al. 2010; Chiou and Bergey 2018). It is likely that this large amount of non-specific exogenous DNA in these samples overwhelmed the small quantity of orangutan DNA in pure un-enriched samples run on the microarray. By decreasing these non-specific DNA concentrations through enrichment, the orangutan DNA was able to be amplified and bind successfully to the homologous human based SNP tagged beads on the microarray. Despite low initial DNA quantities our results corroborate evidence that reliable results can be produced from "near nanogram" levels (Okitsu et al. 2013) on Illumina Infinium SNP microarrays.

Cross species microarray analysis has shown to be possible within species as genetically distant as oryx (Oryx spp.) and modern domesticated bovine (Bos Taurus, e.g., divergent at least 23 million years from each other; Ogden et al. 2012) and Antarctic fur seal (Arctocephalus gazella) and domestic dogs (Canis lupus familiaris) who diverged approximately 44 million years ago (Hoffman et al. 2013). These studies were able to respectively identify 185 of $54,001(0.34 \%)$ and 173 of $173,662(0.01 \%)$ homologous polymorphic loci in common. A further study of wild thin horn (Ovis dalli) and bighorn (Ovis canadensis) sheep genotyped on a chip designed for commercial domestic sheep (Ovis aries; e.g., divergent relatively more recently than the other examples at approximately 3 million years ago) identified 868 of 49,034 loci (1.7\%) to be polymorphic and in common (Miller et al. 2011). Early investigations into ancestral alleles among humans and apes also showed ape DNA can be genotyped using human microarrays (Hacia et al. 1999), and confirmed three and two common homologous polymorphic loci of 397 (0.75\% and $0.5 \%)$ between bonobos and gorillas and humans respectively. Our results producing $125 \mathrm{common}$ genomic polymorphic loci for P. pygmaeus of the 15,949 human loci $(0.78 \%)$ probed for and fit percentages found by these past cross-species studies. These positive results suggest further attempts at cross species genotyping of Pongo DNA on much larger human mapped chips could identify many more common polymorphic SNP loci.

In order to verify the quality of the SNP genotypes produced, microsatellite STR genotyping was conducted for comparison using the Agilent Bioanalyser 2100 through micro-capillary based electrophoretic chips. Despite the fact that resolution of tetrameric STR loci can be problematic on this platform (Fraige et al. 2013) the use of the 2100 Expert software electropherogram overlay and comparison context allowed for calibration of inter-gel and interwell differences. Using repeated amplifications, visual inspection, and known fragment lengths published by previous authors (Utami et al. 2002; Nietlisbach et al. 2010) variants were identifiable within known ranges and genotypes were successfully assigned.

Allele frequencies calculations revealed one marker for the STR dataset and ten SNP loci showing significant deviation from $\mathrm{H}-\mathrm{W}$ equilibrium. Average FIS values across all loci was negative across SNP loci and very close to 0 for STR loci. This negative FIS value in the SNP data suggests an excess of heterozygosity and the possibility of two formerly distinct groups now having admixture. However, more replicates across individuals are needed to confirm SNP assignment and reduce variance in order to help verify this phenomenon. Seven loci (i.e., 1 STR and 6 SNP) also showed a high departure from HW equilibrium could be the result of loss of heterozygosity through inbreeding within the sampled individuals.

Several comparisons of pairwise estimators of relatedness have detailed differences between various statistical methods categorized as either moment and likelihood methods. Wang (2007) compared the TrioML maximum likelihood estimator to several moment estimators including his newest moment estimator (referred to as Wang in Table 4) as well as more commonly used moment estimator by Queller and Goodnight ( $Q$ and $G$ ) and others. This comparison showed that the TrioML produces the most accurate estimates for large datasets both SNP and STR. Subsequent publications have shown

Table 4. Mean sample wide relatedness values for each dataset

\begin{tabular}{lcccccccc}
\hline Dataset & \#of loci & $\mathrm{N}$ & TrioML $(r)$ & var & Wang $(r)$ & var & Q and $G(r)$ & var \\
\hline SNP & 125 & 25 & 0.096 & 0.023 & -0.014 & 0.213 & -0.062 & 0.137 \\
STR & 8 & 29 & 0.082 & 0.020 & 0.008 & 0.097 & -0.041 & 0.072 \\
Combo & 133 & 32 & 0.082 & 0.021 & 0.015 & 0.096 & -0.071 & 0.101 \\
\hline
\end{tabular}

Each dataset, number of loci used and how many individuals were included $n$, each of the three relatedness estimators TrioML, Wang, and Queller and Goodnight $Q$ and $G$, followed by the variance for each estimate 
that in small datasets, where allele frequencies are estimated from the sample for which one is calculating relatedness (as is the case in our dataset), that population wide average moment estimates are expected to be negative and very close to 0 . However, the modified product moment estimators by Wang (2014,2017) and Ritland (Lynch and Ritland 1999) give the least biased pairwise estimates for high and low related individuals respectively. The Wang pairwise relatedness estimated for the known motheroffspring pairs in our dataset indeed appeared to be closest to the expected 0.5 . Through simulations with empirical data Taylor (2015) found that the TrioML estimator correlated closest to "true" relatedness and was least biased for estimates of pairs in high relatedness categories. Thus, it appears that there may be combinations of dataset sizes and types, and estimator types that produce the most accurate estimates for different levels of relatedness. The three relatedness estimators clearly produce quantitatively different sample wide averages in our study across all three of the datasets, with TrioML producing the highest $r$ values, Wang producing a lower and very close to 0 overall $r$, and $Q$ and $G$ producing more negative relatedness values (Table 4 .). These differences in our overall $r$ estimates reflect different estimator biases. The inclusion of further genotyping from more individuals as well as more individuals with known relatedness would help to confirm if this is the case.

However, relatedness estimates calculated using the three estimators in our study were not significantly different between the STR and SNP datasets. Mantel correlation test of matrices of pairwise relatedness estimates for the 22 same individuals showed statistically significant strong positive correlations between $r$ values between any two of the estimators compared. Additionally, we were able to confirm a majority of the known mother-offspring pairs with both datasets. The one mother offspring pair showing lower a relatedness of 0.23 could be due to misidentification of a sample taken with related mothers and offspring in near vicinity during sample collection. Overall, the combined results suggest sufficiently similar pairwise relatedness estimates between both of the SNP and STR genotyping methods and that this method can be used to assign relatedness in unknown individuals and is useful for understand orangutan demographics.

This study verified the existence of a closely related group of wild orangutans including adult males and females who are local to the Camp Leakey Study area. All sampled individuals appear to be from a dispersed but related community. More work will be needed to explicate the relationships in detail. The results from this study show that orangutan DNA collected from feces can provide useful SNP genotypes when run on a human targeted micro-array. Endogenous fecal DNA is often co-extracted with high concentrations of bacterial DNA and inhibitors, thus the Fecalseq enrichment technique was critical to capture endogenous orangutan DNA and reduce inhibitors before conducting SNP analysis. The fact we produced similar relatedness and diversity estimates using this technique provides an exciting new avenue for great ape researchers for discovering SNP loci and genotyping from non-invasive fecal samples from the wild. New genotyping technologies based on the genetic closeness between humans and apes provide opportunities for understanding wild ape populations and providing critical genetic data to support conservation efforts. Any expansion of our knowledge of any of the remaining wild orangutan populations are critical for our overall understanding of the species as a whole and the likelihood of their survival long term.

\section{Conflict of Interest}

The authors declare no conflict of interest.

\section{Acknowledgements}

This study would not have been possible without the support of The Indonesian Ministry of Research, Technology and Higher Education (RISTEKDIKTI), the Indonesian Institute of Sciences (LIPI), The Directorate General of Nature Resources and Ecosystem Conservation, Indonesian Ministry of Environment and Forestry, Tanjung Putting National Park (TNTP), The University of Palangka Raya, Central Kalimantan, and Center for International Cooperation in Sustainable Management of Tropical Peatland (CIMTROP). The authors wish to thank Orangutan Foundation International (OFI) and all the local field assistants associated with Camp Leakey and OFI who helped in sample collection, transport, and processing. This study was funded in part by CWU Graduate and Research office, Graduate Fellowship from CWU GEAR up, Pete and Sandra Barlow Award and Arlen and Debra Prentice Scholarship, as well as Orangutan Foundation International (OFI).

\section{References}

Arora, N., Nater, A., Schaik, C.P. van, Willems, E.P., van Noordwijk, M.A., Goossens, B., Morf, N., Bastian, M., Knott, C., Morrogh-Bernard, H., Kuze, N., Kanamori, T., Pamungkas, J., Dyah Perwitasari-Farajallah, E., Krützen, M., Goodman, M., 2010. Effects of Pleistocene glaciations and rivers on the population structure of Bornean orangutans (Pongo pygmaeus). Proc. Natl. Acad. Sci. U. S. A. 107, 21376-21381. https://doi. org/10.1073/pnas. 1010169107 
Arora, N., van Noordwijk, M.A., Ackermann, C., Willems, E.P., Nater, A., Greminger, M., Nietlisbach, P., Dunkel, L.P., Utami Atmoko, S.S., Pamungkas, J., PerwitasariFarajallah, D., Schaik, C.P. van, Krützen, M., 2012. Parentage-based pedigree reconstruction reveals female matrilineal clusters and male-biased dispersal in nongregarious Asian great apes, the Bornean orangutans (Pongo pygmaeus). Mol. Ecol. 21, 3352-3362.

Bourgeois, S., Mcewing, R., Senn, H., Kaden, J., Taggart, J.B., Ogden, R., Jeffery, K.J., Bunnefeld, N., Abernethy, K., McEwing, R., 2018. Single nucleotide polymorphism discovery and panel characterization in the African forest elephant. Ecol. Evol. 24, 2207-2217. https:// doi.org/10.1002/ece3.3854

Chiou, K.L., Bergey, C.M., 2018. Methylation-based enrichment facilitates low-cost, noninvasive genomic scale sequencing of populations from feces. Sci. Rep. 8, 1975. https://doi.org/10.1038/s41598-018-20427-9

Corless, C.E., Guiver, M., Borrow, R., Edwards-Jones, V. Kaczmarski, E.B., Fox, A.J., 2000. Contamination and sensitivity issues with a real-time universal 16s rRNA PCR. J. Clin. Microbiol. 38, 1747-1752.

Dray, S., Dufour, A.B., 2007. The ade4 package: implementing the duality diagram for ecologists. J. Stat. Softw. 22, 1-20. https://doi.org/10.18637/jss.v022.i04

Fraige, K., Travensolo, R.F., Carrilho, E., 2013. Analysis of seven STR human loci for paternity testing by microchip electrophoresis. Brazilian Arch. Biol. Technol. 56, 213-221. https://doi.org/10.1590/S151689132013000200006

Galdikas, B.M.F., 1979. Orangutan adaptation at Tanjung Puting Reserve: mating and ecology, in: Hamburg, D.A., McCown, E.R.(Eds.), The Great Apes: Perspectives on Human Evolution Volume V. The Benjamin/ Cummings Publishing Company, Menlo Park, Calif, pp. 195-233.

Galdikas, B.M.F., 1982. Orangutan tool-use at Tanjung Puting Reserve, central Indonesian Borneo (Kalimantan Tengah). J. Hum. Evol. 10, 19-33.

Galdikas, B.M.F., 1985. Orangutan sociality at Tanjung Puting. Am. J. Primatol. 9, 101-119.

Galdikas, B.M.F., 1988. Orangutan diet, range, and activity at Tanjung Puting, Central Borneo. Int. J. Primatol. 9, 1-35. https://doi.org/10.1007/BF02740195

Goossens, B., Chikhi, L., Utami, S.S., de Ruiter, J., Bruford, M.W., 2000. A multi-samples, multi-extracts approach for microsatellite analysis of faecal samples in an arboreal ape. Conserv. Genet. 1, 157-162. https://doi. org/10.1023/a:1026535006318

Goossens, B., Chikhi, L., Jalil, M.F., Ancrenaz, M., LackmanAncrenaz, I., Mohamed, M., Andau, P., Bruford, M.W. 2005. Patterns of genetic diversity and migration in increasingly fragmented and declining orang-utan (Pongo pygmaeus) populations from Sabah, Malaysia. Mol. Ecol. 14, 441-456.

Goossens, B., Setchell, J.M., James, S.S., Funk, S.M., Chikhi, L., Abulani, A., Ancrenaz, M., Lackman-Ancrenaz, I., Bruford, M.W., 2006. Philopatry and reproductive success in Bornean orang-utans (Pongo pygmaeus). Molecular Ecology, 15, 2577-2588. https://doi. org/10.1111/j.1365-294X.2006.02952.x

Hacia, J.G., Fan, J.B., Ryder, O., Jin, L., Edgemon, K., Ghandour, G., Mayer, R.A., Sun, B., Hsie, L., Robbins, C.M., Brody, L.C., Wang, D., Lander, E.S., Lipshutz, R., Fodor, S.P.A., Collins, F.S., 1999. Determination of ancestral alleles for human single-nucleotide polymorphisms using high-density oligonucleotide arrays. Nat. Genet. 22, 164-167. https://doi.org/10.1038/9674

Higuchi, R., Fockler, C., Dollinger, G., Watson, R., 1993. Kinetic PCR analysis: real-time monitoring of DNA amplification reactions. Bio/Technology 11, 10261030. https://doi.org/10.1038/nbt0993-1026
Hoffman, J.I., Thorne, M.A.S., McEwing, R., Forcada, J., Ogden, R., 2013. Cross-amplification and validation of SNPs conserved over 44 million years between seals and dogs. PLoS One 8, 1-10. https://doi.org/10.1371/ journal.pone.0068365

Inoue, E., Inoue-Murayama, M., Takenaka, O., Nishida, T., 2007. Wild chimpanzee infant urine and saliva sampled noninvasively usable for DNA analyses. Primates 48 , 156-159. https://doi.org/10.1007/s10329-006-0017-y

Jones, O.R., Wang, J., 2010. COLONY: a program for parentage and sibship inference from multilocus genotype data. Mol. Ecol. Resour. 10, 551-555. https://doi. org/10.1111/j.1755-0998.2009.02787.x

Kalinowski, S.T., Taper, M.L., Marshall, T.C., 2007. Revising how the computer program CERVUS accommodates genotyping error increases success in paternity assignment. Mol. Ecol. 16, 1099-1106. https://doi. org/10.1111/j.1365-294X.2007.03089.X

Kanthaswamy, S., Capitanio, J.P., Dubay, C.J., Ferguson, B., Folks, T., Ha, J.C., 2009. Resources for genetic management and genomics research on non-human primates at the National Primate Research Centers (NPRCs). J. Med. Primatol. 38, 17-23. https://doi. org/10.1111/j.1600-0684.2009.00371.x

Krawczak, M., 1999. Informativity assessment for biallelic single nucleotide polymorphisms. Electrophoresis. 20, 1676-1681.

Lynch, M., Ritland, K., 1999. Estimation of pairwise relatedness with molecular markers. Tbe Genet. Soc. Am. 152, 1753-1766.

Miller, J.M., Poissant, J., Kijas, J.W., Coltman, D.W., 2011. A genome-wide set of SNPs detects population substructure and long range linkage disequilibrium in wild sheep. Mol. Ecol. Resour. 11, 314-322.

Morrogh-Bernard, H.C., Morf, N.V., Chivers, D.J., Krützen, M. 2011. Dispersal patterns of orang-utans (Pongo spp.) in a Bornean peat-swamp forest. Int. J. Primatol. 32, 362-376.

Nietlisbach, P., Nater, A., Greminger, M.P., Arora, N., Krützen, M., 2010. A multiplex-system to target 16 male-specific and 15 autosomal genetic markers for orang-utans (Genus: Pongo). Conserv. Genet. Resour. 2, 153-158.

Norman, A.J., Street, N.R., Spong, G., 2013. De novo SNP discovery in the scandinavian brown bear (Ursus arctos). PLoS One 8, 1-12. https://doi.org/10.1371/ journal.pone.0081012

Nsubuga, A.M., Robbins, M.M., Roeder, A.D., Morin, P.A., Boesch, C., Vigilant, L., 2004. Factors affecting the amount of genomic DNA extracted from ape faeces and the identification of an improved sample storage method. Mol. Ecol. 13, 2089-2094. https://doi. org/10.1111/j.1365-294X.2004.02207.x

Ogden, R., Baird, J., Senn, H., McEwing, R., 2012. The use of cross-species genome-wide arrays to discover SNP markers for conservation genetics: A case study from Arabian and scimitar-horned oryx. Conserv. Genet. Resour. 4, 471-473.

Okitsu, C.Y., Berg, D.J. Van Den, Lieber, M.R., Hsieh, C., 2013. Reproducibility and reliability of SNP analysis using human cellular DNA at or near nanogram levels. BMC Res. Notes 6, 1-6.

Perry, G.H., Marioni, J.C., Melsted, P., Gilad, Y., 2010. Genomicscale capture and sequencing of endogenous DNA from feces. Mol. Ecol. 19, 5332-5344.

Queller, D.C., Goodnight, K.F., 1989. Estimating relatedness using genetic markers. Evolution. 43, 258-275.

R Core Team, 2014. R: a language and environment for statistical computing. R Found. Stat. Comput. Vienna, Austria. Available at: http//www.R-project.org/. [Date accessed: 4 December 2019] 
Rianti, P., Perwitasari-Farajallah, D., Sajuthi, D., Pamungkas, J., Nater, A., Krützen, M., 2015. Identification of diagnostic mitochondrial DNA single nucleotide polymorphisms specific to Sumatran Orangutan (Pongo abelii) populations. HAYATI J. Biosci. 22, 149156. https://doi.org/10.1016/j.hjb.2015.09.002

Ross, C.T., Weise, J.A., Bonnar, S., Nolin, D., Satkoski Trask, J., Smith, D.G., Ferguson, B., Ha, J., Kubisch, H.M., Vinson, A., Kanthaswamy, S., 2014. An empirical comparison of short tandem repeats (STRs) and single nucleotide polymorphisms (SNPs) for relatedness estimation in Chinese rhesus macaques (Macaca mulatta). Am. J. Primatol. 76, 313-324.

Rousset, F., 2008. Genepop'007: a complete re-implementation of the genepop software for Windows and Linux. Mol. Ecol. Resour. 8, 103-106. https://doi.org/10.1111/ j.1471-8286.2007.01931.x

Rutledge, L.Y., Holloway, J.J., Patterson, B.R., White, B.N., 2009. An improved field method to obtain DNA for individual identification from wolf scat. J. Wildl. Manage. 73, 1430-1435.

Simons, N.D., Lorenz, J.G., Sheeran, L.K., Li, J.H., Xia, D.P., Wagner, R.S., 2012. Noninvasive saliva collection for DNA analyses from free-ranging Tibetan Macaques (Macaca thibetana). American Journal of Primatology. 74, 1064-1070. https://doi.org/10.1002/ajp.22062

Taylor, H. R. 2015. The use and abuse of genetic marker-based estimates of relatedness and inbreeding. Ecology and Evolution. 5, 3140-3150. https://doi.org/10.1002/ ece 3.1541

Utami, S.S., Goossens, B., Bruford, M.W., De Ruiter, J.R., van Hooff, J.a.R.a.M., 2002. Male bimaturism success and reproductive success in Sumatran orang-utans. Behav. Ecol. 13, 643-652.
Utami-Atmoko, S.S., Traylor-Holzer, K., Rifqi, M.A., Siregar, P.G., Achmad, B., Priadjati, A., Husson, S., Wich, S., Hadisiswoyo, P., Saputra, F., Campbell-Smith, G., Kuncoro, P., Russon, A., Voigt, M., Santika, T., Nowak, M., Singleton, I., Sapari, I., Meididit, A., Chandradewi, D.S., Ripoll Capilla, B., Ermayanti, Lees, C.M., 2017. Orangutan population and habitat viability assessment: final report. IUCN/SSC Conservation Breeding Specialist Group, Apple Valley, MN.

Vigilant, L., Guschanski, K., 2009. Using genetics to understand the dynamics of wild primate populations. Primates 50, 105-120. https://doi.org/10.1007/s10329-0080124-Z

Wang, J., 2002. An estimator for pairwise relatedness using molecular markers. Genetics 160, 1203-1215.

Wang, J., 2007. Triadic IBD coefficients and applications to estimating pairwise relatedness. Genet. Res. 89, 135153. https://doi.org/10.1017/S0016672307008798

Wang, J., 2011. Coancestry: a program for simulating, estimating and analysing relatedness and inbreeding coefficients. Mol. Ecol. Resour. 11, 141-145. https:// doi.org/10.1111/j.1755-0998.2010.02885.x

Wang, J., 2014. Marker-based estimates of relatedness and inbreeding coefficients: an assessment of current methods. J. Evol. Biol. 27, 518-530. https://doi. org/10.1111/jeb.12315

Wang, J., 2017. Estimating pairwise relatedness in a small sample of individuals. Nat. Publ. Gr. 119, 302-313. https://doi.org/10.1038/hdy.2017.52

Weir, B.S., Cockerham, C.C., 1984. Estimating F-statistics for the analysis of population structure. Evolution (N. Y). 38, 1358-1370. https://doi.org/10.1111/j.1558-5646.1984. tb05657.x 
Supplementary S1. SNP Loci

Di-allelic human SNP loci, human chromosome number and MapInfo coordinate for SNP, number of individuals genotyped, observed heterozygosity HO and expected heterozygosity HE, polymorphic information content PIC, p-value of probability test for deviation from Hardy-Weinberg equilibrium, and average FIS (Weir and Cockerham 1984)

\begin{tabular}{|c|c|c|c|c|c|c|c|c|c|}
\hline SNP name & $\begin{array}{l}\text { Human } \\
\text { chrom }\end{array}$ & Coordinate & $n$ & $\mathrm{H}_{\mathrm{o}}$ & $\mathrm{H}_{\mathrm{E}}$ & PIC & P-val & S.E. & $\mathrm{F}_{\mathrm{IS}}$ Wa and $\mathrm{C}$ \\
\hline$\overline{1: 25617206-\mathrm{CT}}$ & 1 & 25290715 & 25 & 0.16 & 0.15 & 0.136 & 1.000 & 0.000 & -0.067 \\
\hline 1:25729163-GA & 1 & 25402672 & 23 & 0.04 & 0.04 & 0.042 & \multicolumn{3}{|c|}{ No information } \\
\hline 19:49206962-GA & 19 & 48703705 & 24 & 0.13 & 0.19 & 0.169 & 0.207 & 0.002 & 0.349 \\
\hline 19:579157-TC & 19 & 579157 & 20 & 0.45 & 0.45 & 0.342 & 1.000 & 0.000 & 0.000 \\
\hline 6:31239829-СТ & 6 & 31272052 & 20 & 0.35 & 0.30 & 0.247 & 1.000 & 0.000 & -0.188 \\
\hline 6:32610134-GA & 6 & 32642357 & 20 & 0.55 & 0.45 & 0.342 & 0.607 & 0.002 & -0.229 \\
\hline 9:136131022-C-T & 9 & 133255635 & 17 & 0.88 & 0.52 & 0.375 & $0.004^{*}$ & 0.000 & -0.752 \\
\hline 9:136131415-CT & 9 & 133256028 & 20 & 0.55 & 0.48 & 0.359 & 0.646 & 0.002 & -0.148 \\
\hline 9:136137555-G-A & 9 & 133262152 & 22 & 0.09 & 0.24 & 0.208 & $0.025^{*}$ & 0.001 & 0.628 \\
\hline 9:136146449-TAAGAC-T & 9 & 133271018 & 10 & 0.70 & 0.48 & 0.351 & 0.221 & 0.002 & -0.500 \\
\hline 9:139925644-GA & 9 & 137031192 & 20 & 0.30 & 0.26 & 0.222 & 1.000 & 0.000 & -0.152 \\
\hline 9:139925843-CA & 9 & 137031391 & 19 & 0.37 & 0.31 & 0.255 & 1.000 & 0.000 & -0.200 \\
\hline exm2229707 & 19 & 48596811 & 18 & 0.44 & 0.51 & 0.375 & 0.655 & 0.002 & 0.139 \\
\hline exm224876 & 2 & 126696000 & 19 & 0.47 & 0.46 & 0.349 & 1.000 & 0.000 & -0.025 \\
\hline exm2260060 & 1 & 240579605 & 20 & 0.55 & 0.41 & 0.319 & 0.256 & 0.002 & -0.357 \\
\hline exm2260204 & 13 & 41549067 & 19 & 0.16 & 0.15 & 0.135 & 1.000 & 0.000 & -0.059 \\
\hline exm2260552 & 16 & 50669787 & 21 & 0.14 & 0.14 & 0.124 & 1.000 & 0.000 & -0.053 \\
\hline exm2261221 & 2 & 236300554 & 14 & 0.43 & 0.35 & 0.280 & 1.000 & 0.000 & -0.238 \\
\hline exm2261348 & 3 & 10664912 & 17 & 0.35 & 0.30 & 0.248 & 1.000 & 0.000 & -0.185 \\
\hline exm2262610 & 9 & 137345126 & 18 & 0.17 & 0.25 & 0.211 & 0.274 & 0.002 & 0.329 \\
\hline exm2264375 & 9 & 138066115 & 21 & 0.24 & 0.22 & 0.188 & 1.000 & 0.000 & -0.111 \\
\hline exm 2265018 & 1 & 74396083 & 20 & 0.25 & 0.51 & 0.374 & 0.030 & 0.001 & 0.518 \\
\hline exm2265648 & 3 & 188976960 & 16 & 0.44 & 0.42 & 0.323 & 1.000 & 0.000 & -0.050 \\
\hline exm2266502 & 7 & 5793154 & 13 & 0.39 & 0.32 & 0.262 & 1.000 & 0.000 & -0.200 \\
\hline exm2266554 & 7 & 101448189 & 25 & 0.32 & 0.27 & 0.233 & 1.000 & 0.000 & -0.171 \\
\hline exm2267112 & 10 & 81963701 & 6 & 0.33 & 0.55 & 0.375 & 0.476 & 0.002 & 0.412 \\
\hline exm2267114 & 10 & 84338568 & 16 & 0.50 & 0.48 & 0.359 & 1.000 & 0.000 & -0.035 \\
\hline exm2268218 & 19 & 12581187 & 23 & 0.61 & 0.46 & 0.351 & 0.180 & 0.002 & -0.322 \\
\hline exm2269623 & 3 & 51378937 & 21 & 0.33 & 0.29 & 0.239 & 1.000 & 0.000 & -0.177 \\
\hline exm2270539 & 6 & 161727303 & 21 & 0.62 & 0.44 & 0.336 & 0.116 & 0.002 & -0.429 \\
\hline exm2271402 & 10 & 55891194 & 19 & 0.16 & 0.15 & 0.135 & 1.000 & 0.000 & -0.059 \\
\hline exm2271881 & 12 & 129933255 & 15 & 0.47 & 0.37 & 0.294 & 0.530 & 0.002 & -0.273 \\
\hline exm2272151 & 14 & 20349972 & 21 & 0.24 & 0.22 & 0.188 & 1.000 & 0.000 & -0.111 \\
\hline exm2272325 & 15 & 90960641 & 15 & 0.20 & 0.19 & 0.164 & 1.000 & 0.000 & -0.077 \\
\hline exm2272572 & 17 & 8124275 & 13 & 0.31 & 0.27 & 0.226 & 1.000 & 0.000 & -0.143 \\
\hline exm51163 & 1 & 42830512 & 21 & 0.29 & 0.48 & 0.360 & 0.077 & 0.002 & 0.415 \\
\hline exm518984 & 6 & 18143724 & 11 & 0.36 & 0.52 & 0.373 & 0.540 & 0.002 & 0.310 \\
\hline exm526563 & 6 & 29828746 & 19 & 0.26 & 0.24 & 0.202 & 1.000 & 0.000 & -0.125 \\
\hline exm537081 & 6 & 32938875 & 19 & 0.21 & 0.19 & 0.171 & 1.000 & 0.000 & -0.091 \\
\hline exm537383 & 6 & 33068728 & 21 & 0.24 & 0.29 & 0.239 & 0.451 & 0.002 & 0.167 \\
\hline exm537454 & 6 & 33069863 & 21 & 0.62 & 0.47 & 0.354 & 0.188 & 0.002 & -0.327 \\
\hline exm537513 & 6 & 33080851 & 18 & 0.33 & 0.36 & 0.286 & 1.000 & 0.000 & 0.064 \\
\hline exm612728 & 7 & 30922175 & 20 & 0.15 & 0.14 & 0.129 & 1.000 & 0.000 & -0.056 \\
\hline exm-rs3117034 & 6 & 33119581 & 19 & 0.21 & 0.27 & 0.231 & 0.371 & 0.002 & 0.234 \\
\hline exm-rs8176746 & 9 & 133255935 & 22 & 0.68 & 0.50 & 0.370 & 0.186 & 0.002 & -0.370 \\
\hline JHU_1.3691239 & 1 & 3774676 & 20 & 0.20 & 0.26 & 0.222 & 0.352 & 0.002 & 0.240 \\
\hline JHU_11.35177589 & 11 & 35156043 & 19 & 0.16 & 0.15 & 0.135 & 1.000 & 0.000 & -0.059 \\
\hline JHU_11.35216457 & 11 & 35194911 & 11 & 0.27 & 0.52 & 0.375 & 0.220 & 0.002 & 0.492 \\
\hline JHU_17.42329003 & 17 & 44251636 & 24 & 0.29 & 0.31 & 0.258 & 1.000 & 0.000 & 0.064 \\
\hline JHU_17.42330696 & 17 & 44253329 & 20 & 0.15 & 0.14 & 0.129 & 1.000 & 0.000 & -0.056 \\
\hline JHU_2.127436468 & 2 & 126678893 & 11 & 0.18 & 0.17 & 0.152 & 1.000 & 0.000 & -0.053 \\
\hline JHU_22.43100132 & 22 & 42704127 & 23 & 0.22 & 0.20 & 0.175 & 1.000 & 0.000 & -0.100 \\
\hline JHU_6.10535520 & 6 & 10535288 & 19 & 0.21 & 0.27 & 0.231 & 0.374 & 0.002 & 0.234 \\
\hline JHU_6.10535603 & 6 & 10535371 & 20 & 0.10 & 0.10 & 0.090 & 1.000 & 0.000 & -0.027 \\
\hline JHU_6.32607324 & 6 & 32639548 & 18 & 0.44 & 0.36 & 0.286 & 0.526 & 0.002 & -0.259 \\
\hline
\end{tabular}


Supplementary S1. Continued

\begin{tabular}{|c|c|c|c|c|c|c|c|c|c|}
\hline SNP name & $\begin{array}{l}\text { Human } \\
\text { chrom }\end{array}$ & Coordinate & $n$ & $\mathrm{H}_{\mathrm{o}}$ & $\mathrm{H}_{\mathrm{E}}$ & PIC & P-val & S.E. & $\mathrm{F}_{\mathrm{IS}}$ Wa and C \\
\hline JHU_6.32607610 & 6 & 32639834 & 19 & 0.63 & 0.48 & 0.357 & 0.315 & 0.003 & -0.333 \\
\hline JHU_6.32608034 & 6 & 32640258 & 16 & 0.81 & 0.50 & 0.366 & $0.014^{*}$ & 0.001 & -0.667 \\
\hline JHU_6.32608356 & 6 & 32640580 & 16 & 0.31 & 0.27 & 0.229 & 1.000 & 0.000 & -0.154 \\
\hline JHU_6.32610682 & 6 & 32642906 & 17 & 0.35 & 0.30 & 0.248 & 1.000 & 0.000 & -0.185 \\
\hline JHU_6.32628305 & 6 & 32660529 & 15 & 0.07 & 0.43 & 0.332 & $0.002^{*}$ & 0.000 & 0.851 \\
\hline JHU_6.32629270 & 6 & 32661494 & 17 & 0.65 & 0.51 & 0.372 & 0.347 & 0.002 & -0.285 \\
\hline JHU_6.32629370 & 6 & 32661594 & 17 & 0.35 & 0.50 & 0.367 & 0.324 & 0.002 & 0.299 \\
\hline JHU_6.32629548 & 6 & 32661772 & 21 & 0.67 & 0.51 & 0.374 & 0.205 & 0.003 & -0.315 \\
\hline JHU_6.32629602 & 6 & 32661826 & 20 & 0.70 & 0.51 & 0.372 & 0.172 & 0.002 & -0.393 \\
\hline JHU_6.32629617 & 6 & 32661841 & 14 & 0.21 & 0.50 & 0.363 & 0.085 & 0.002 & 0.576 \\
\hline JHU_6.32629679 & 6 & 32661903 & 17 & 0.82 & 0.50 & 0.367 & $0.010^{*}$ & 0.001 & -0.684 \\
\hline JHU_6.32630966 & 6 & 32663190 & 19 & 0.47 & 0.46 & 0.349 & 1.000 & 0.000 & -0.025 \\
\hline JHU_6.32633225 & 6 & 32665449 & 16 & 0.56 & 0.50 & 0.366 & 1.000 & 0.000 & -0.135 \\
\hline JHU_6.33032864 & 6 & 33065088 & 14 & 0.64 & 0.45 & 0.341 & 0.220 & 0.002 & -0.444 \\
\hline JHU_6.33045658 & 6 & 33077882 & 21 & 0.24 & 0.22 & 0.188 & 1.000 & 0.000 & -0.111 \\
\hline JHU_6.33053788 & 6 & 33086012 & 15 & 0.47 & 0.43 & 0.332 & 1.000 & 0.000 & -0.077 \\
\hline JHU_6.33089374 & 6 & 33121598 & 19 & 0.21 & 0.27 & 0.231 & 0.369 & 0.002 & 0.234 \\
\hline kgp13606542 & 7 & 95295992 & 16 & 0.38 & 0.39 & 0.305 & 1.000 & 0.000 & 0.032 \\
\hline kgp15099441 & 22 & 42129132 & 13 & 0.15 & 0.27 & 0.226 & 0.235 & 0.002 & 0.442 \\
\hline kgp3038063 & 16 & 89553920 & 19 & 0.21 & 0.19 & 0.171 & 1.000 & 0.000 & -0.091 \\
\hline kgp451798 & 10 & 72013241 & 12 & 0.67 & 0.52 & 0.375 & 0.563 & 0.002 & -0.294 \\
\hline kgp9521982 & 8 & 69832577 & 18 & 0.39 & 0.32 & 0.264 & 1.000 & 0.000 & -0.214 \\
\hline rs1000709 & 9 & 114475474 & 20 & 0.20 & 0.19 & 0.164 & 1.000 & 0.000 & -0.086 \\
\hline rs1034063 & 20 & 3051186 & 23 & 0.44 & 0.43 & 0.334 & 1.000 & 0.000 & -0.005 \\
\hline rs1042544 & 6 & 33086680 & 12 & 0.42 & 0.43 & 0.328 & 1.000 & 0.000 & 0.035 \\
\hline rs1055055 & 20 & 5546645 & 10 & 0.30 & 0.27 & 0.222 & 1.000 & 0.000 & -0.125 \\
\hline rs1058433 & 1 & 93154836 & 17 & 0.12 & 0.30 & 0.248 & $0.042^{*}$ & 0.001 & 0.615 \\
\hline rs1060622 & 9 & 37974746 & 17 & 0.71 & 0.47 & 0.352 & $0.049^{*}$ & 0.001 & -0.524 \\
\hline rs1138374 & 20 & 16260771 & 25 & 0.24 & 0.49 & 0.365 & $0.014^{*}$ & 0.001 & 0.515 \\
\hline rs12480506 & 3 & 160086741 & 11 & 0.55 & 0.52 & 0.373 & 1.000 & 0.000 & -0.053 \\
\hline rs12634498 & 1 & 151874041 & 22 & 0.00 & 0.09 & 0.083 & $0.024^{*}$ & 0.001 & 1.000 \\
\hline rs13320 & 5 & 102335711 & 19 & 0.63 & 0.50 & 0.369 & 0.358 & 0.003 & -0.271 \\
\hline rs1584717 & 13 & 28734932 & 8 & 0.63 & 0.53 & 0.371 & 1.000 & 0.000 & -0.207 \\
\hline rs1617234 & 6 & 29747985 & 16 & 0.38 & 0.52 & 0.375 & 0.341 & 0.002 & 0.280 \\
\hline rs1633086 & 11 & 66504671 & 21 & 0.00 & 0.09 & 0.087 & $0.024^{*}$ & 0.001 & 1.000 \\
\hline rs1671063 & 7 & 87504154 & 17 & 0.18 & 0.17 & 0.148 & 1.000 & 0.000 & -0.067 \\
\hline rs17064 & 7 & 130331348 & 19 & 0.21 & 0.19 & 0.171 & 1.000 & 0.000 & -0.091 \\
\hline rs1760921 & 17 & 12747460 & 21 & 0.29 & 0.25 & 0.215 & 1.000 & 0.000 & -0.143 \\
\hline rs1800462 & 22 & 29232752 & 12 & 0.50 & 0.51 & 0.368 & 1.000 & 0.000 & 0.015 \\
\hline rs1809627 & 12 & 129872147 & 19 & 0.37 & 0.37 & 0.296 & 1.000 & 0.000 & 0.008 \\
\hline rs1980889 & 2 & 240419949 & 17 & 0.18 & 0.17 & 0.148 & 1.000 & 0.000 & -0.067 \\
\hline rs1984661 & 3 & 193682987 & 21 & 0.05 & 0.05 & 0.045 & \multicolumn{3}{|c|}{ No information } \\
\hline rs1997719 & 14 & 106852518 & 17 & 0.59 & 0.50 & 0.367 & 0.624 & 0.002 & -0.185 \\
\hline rs2047709 & 6 & 33007734 & 19 & 0.53 & 0.44 & 0.339 & 0.610 & 0.002 & -0.192 \\
\hline rs2078402 & 17 & 50683744 & 18 & 0.61 & 0.48 & 0.355 & 0.321 & 0.002 & -0.299 \\
\hline rs2088335 & 16 & 2895089 & 16 & 0.56 & 0.42 & 0.323 & 0.257 & 0.002 & -0.364 \\
\hline rs2105992 & 15 & 25197251 & 20 & 0.05 & 0.05 & 0.048 & \multicolumn{3}{|c|}{ No information } \\
\hline rs2267647 & 19 & 15929482 & 19 & 0.21 & 0.34 & 0.277 & 0.143 & 0.002 & 0.390 \\
\hline rs2277624 & 6 & 6168985 & 25 & 0.24 & 0.27 & 0.233 & 0.485 & 0.002 & 0.127 \\
\hline rs2301763 & 5 & 601532 & 22 & 0.23 & 0.21 & 0.181 & 1.000 & 0.000 & -0.105 \\
\hline rs2739765 & 10 & 5899990 & 22 & 0.27 & 0.30 & 0.253 & 0.539 & 0.002 & 0.106 \\
\hline rs3740066 & 6 & 32938451 & 12 & 0.42 & 0.43 & 0.328 & 1.000 & 0.000 & 0.035 \\
\hline rs3765070 & 11 & 69261743 & 18 & 0.22 & 0.20 & 0.178 & 1.000 & 0.000 & -0.097 \\
\hline rs3823193 & 4 & 21094522 & 18 & 0.11 & 0.11 & 0.099 & 1.000 & 0.000 & -0.030 \\
\hline rs3828570 & 19 & 17366509 & 21 & 0.48 & 0.42 & 0.325 & 0.631 & 0.002 & -0.143 \\
\hline rs591510 & 17 & 61596942 & 18 & 0.61 & 0.44 & 0.334 & 0.120 & 0.002 & -0.417 \\
\hline rs6934645 & 1 & 5335808 & 16 & 0.25 & 0.23 & 0.195 & 1.000 & 0.000 & -0.111 \\
\hline rs7122786 & 22 & 42716955 & 20 & 0.40 & 0.47 & 0.351 & 0.633 & 0.002 & 0.146 \\
\hline
\end{tabular}


Supplementary S1. Continued

\begin{tabular}{|c|c|c|c|c|c|c|c|c|c|}
\hline SNP name & $\begin{array}{l}\text { Human } \\
\text { chrom }\end{array}$ & Coordinate & $n$ & $\mathrm{H}_{\mathrm{O}}$ & $\mathrm{H}_{\mathrm{E}}$ & PIC & P-val & S.E. & $\mathrm{F}_{\mathrm{IS}}$ Wa and C \\
\hline rs720853 & 10 & 124648781 & 20 & 0.60 & 0.49 & 0.365 & 0.385 & 0.003 & -0.226 \\
\hline rs7248564 & 10 & 133239619 & 17 & 0.29 & 0.26 & 0.219 & 1.000 & 0.000 & -0.143 \\
\hline rs725900 & 6 & 33082268 & 13 & 0.46 & 0.49 & 0.361 & 1.000 & 0.000 & 0.065 \\
\hline rs729206 & 6 & 33087470 & 13 & 0.54 & 0.41 & 0.316 & 0.500 & 0.002 & -0.333 \\
\hline rs738527 & 6 & 33088972 & 23 & 0.26 & 0.23 & 0.201 & 1.000 & 0.000 & -0.128 \\
\hline rs876352 & 21 & 43283415 & 21 & 0.14 & 0.22 & 0.188 & 0.232 & 0.002 & 0.341 \\
\hline rs880340 & 1 & 42414845 & 17 & 0.29 & 0.40 & 0.314 & 0.527 & 0.002 & 0.273 \\
\hline rs907100 & 14 & 20349972 & 15 & 0.27 & 0.24 & 0.204 & 1.000 & 0.000 & -0.120 \\
\hline rs9277361 & 6 & 18143724 & 13 & 0.54 & 0.41 & 0.316 & 0.502 & 0.002 & -0.333 \\
\hline rs9277542 & 9 & 89284330 & 17 & 0.59 & 0.47 & 0.352 & 0.589 & 0.002 & -0.260 \\
\hline rs9277561 & 10 & 99844450 & 24 & 0.38 & 0.40 & 0.317 & 1.000 & 0.000 & 0.072 \\
\hline \multirow[t]{3}{*}{ rs976531 } & 2 & 238654938 & 18 & 0.39 & 0.32 & 0.264 & 1.000 & 0.000 & -0.214 \\
\hline & & Average & 18 & 0.36 & 0.34 & 0.268 & 0.658 & 0.001 & -0.037 \\
\hline & & SD & & 0.19 & 0.14 & 0.092 & & & 0.308 \\
\hline
\end{tabular}

*indicates significant deviation from HW equilibrium 